\title{
Use of recombinant human growth hormone in alveolar ridge preservation procedures: a case
} report

\begin{abstract}
Recombinant Human Growth Hormone (RhGh) has been studied in cases of dental implants, TMJ pathologies and bone fractures. It is able to stimulate bone growth in a dose-dependent manner by direct stimulation of chondrocytes. It stimulates proliferation and differentiation of chondroprogenitor cells, also acts directly on osteoblasts, increasing rates of remodeling and bone formation. In this review of the literature and case report, RhGh will be used in alveolar ridge preservation procedures, with the purpose of evaluating the benefits of this technique in maintaining the post-extraction bone site viable, aiming a future rehabilitation with dental implants and prostheses.
\end{abstract}

Keywords: human growth hormone, alveolar process, tooth extraction, maxilla
Volume 8 Issue 4 - 2017

\author{
Leonardo Matos Santolim Zanettini,' \\ Ricardo Giacomini de Marco,' Fernando \\ de Oliveira Andriola,' Fernando Antonini,' \\ Helena Wilhelm de Oliveira, ${ }^{2}$ Rogerio \\ Miranda Pagnoncelli' \\ 'Department of Oral and Maxillofacial Surgery, Pontifical \\ Catholic University of Rio Grande do Sul (PUCRS), Brazil \\ ${ }^{2}$ Department of Radiology, Pontifical Catholic University of Rio \\ Grande do Sul (PUCRS), Brazil
}

\begin{abstract}
Correspondence: Leonardo Matos Santolim Zanettini, Student of the Master's Program in Oral and Maxillofacial Surgery, Pontifical Catholic University of Rio Grande do Sul (PUCRS):Av. Ipiranga, 668I, Partenon 90619-900, Porto Alegre, Rio Grande do Sul, Brazil, Email leonardo.zanettini@acad.pucrs.br
\end{abstract}

Received: August 10,2017 | Published: October 17, 2017
Abbreviations: RhGh, recombinant human growth hormone; $\mathrm{CBCT}$, cone beam computed tomography; UI, international unites; $\mathrm{GH}$, growth hormone.

\section{Introduction}

The alveolar processes of the jaws are tooth-dependent structures that undergo significant structural losses when some dental organ is lost. The dynamics and magnitude of these changes have already been investigated in animal and human study models. These investigations identified the key to the process of bone remodeling after tooth extraction, which promotes the reduction and significant alterations in the dimensions of the alveolar ridges. ${ }^{1-3}$ Studies show that the most severe resorption occurs in the first 3 months of healing, although dimensional changes can be observed after one year of tooth extraction, resulting in a reduction of approximately $50 \%$ in the buccal-lingual / palatal dimension of the alveolus. ${ }^{4}$ The clinical consequences of these physiological tissue changes may affect the results of therapies used to restore lost dentition, limiting the optimal bone quantity for implant placement or even compromising aesthetic results for prosthesis rehabilitation. Different therapies of alveolar preservation were proposed, such as: minimally invasive surgeries, without flaps; Dental extractions and immediate implants; filling the alveolus with different types of graft materials, with or without membrane. ${ }^{5}$ Alveolar preservation therapies were proposed with the objective of maintaining the dimensions of alveolar bone tissue, which would be lost after the dental extraction procedures, as part of a natural physiological process of healing. The purpose of these therapies is to prepare the bone site for the placement of osseointegrated implants, in order to have a viable bone for rehabilitation. ${ }^{6}$ Recombinant Human Growth Hormone (RhGh) has been studied in cases of dental implants, ${ }^{7}$ use in TMJ pathologies, and bone fractures. ${ }^{8,9}$ Human Growth Hormone is produced by the anterior pituitary gland and has an effect on all cells of the body, increasing the speed of protein synthesis, increasing the consumption of fatty acids and reducing the speed of glucose consumption. In this way, it preserves carbohydrates, consumes reserves of energy and increases the deposition of proteins, including the chondrocytic and osteogenic cells. ${ }^{10} \mathrm{RhGH}$ is capable of stimulating bone growth in a dose-dependent manner by direct stimulation of chondrocytes. It stimulates the proliferation and differentiation of chondroprogenitor cells. It also acts directly on osteoblasts, increasing remodeling rates and bone formation. ${ }^{11}$ Based on the above evidence, the purpose of this article was to report a case using Recombinant Human Growth Hormone in post-extraction alveolar preservation procedures, in order to evaluate the benefits of this technique, which is promising to maintain the post-extraction bone site viable, aiming the future rehabilitation with dental implants and prostheses.

\section{Case presentation}

A 39-year-old patient was seen at the Oral and Maxillofacial Surgery service at Pontifical Catholic University of Rio Grande do Sul (PUCRS) with functional and esthetic complaints. Intra-oral clinical examination, cone beam computed tomography (CBCT) and facial analysis found the patient to have missing teeth $(13,14,15,16)$, esthetic problems with the reminiscent teeth $(12,11,21,22,23,24$, $25,26,27,28)$, root resorption of the anterior teeth, malpositioned teeth and Class III occlusion (Figures $1 \& 2$ ). The risks and benefits of many alternative of dental treatment were evaluated and chosen option recommended to correct the functional and esthetic patient complaints was the extraction of the all the remaining teeth aiming the placement of dental implants and a restoration with a fixed prosthesis. Surgical planning included CBCT, facial and models analysis. The surgical plan defined a two time procedure. First, the extraction of the remaining teeth and placement of 2 UI of Somatropin on each post- 
extraction sites aiming to maintain the dimensions of alveolar bone tissue. After 3-4 months healing, it will be planed the implants surgery. The surgery was performed under local anesthesia (Articaine 4\% 1: 100.000). The basic requirements for oral surgery were observed, such as minimally invasive surgery, using periotomes, without flap, in order to decrease the process of alveolar bone resorption. The dental elements 17 and 27 were maintained in order to preserve the patient occlusal vertical dimension (OVD). During the healing process, a removable partial denture was performed. The patient was followedup weekly. Antibiotic therapy and analgesia were prescribed, and also control computed tomography was conducted immediate after surgery, 30 days after healing and a new CBCT will be performed after 3-4 months healing (Figures 3-7).

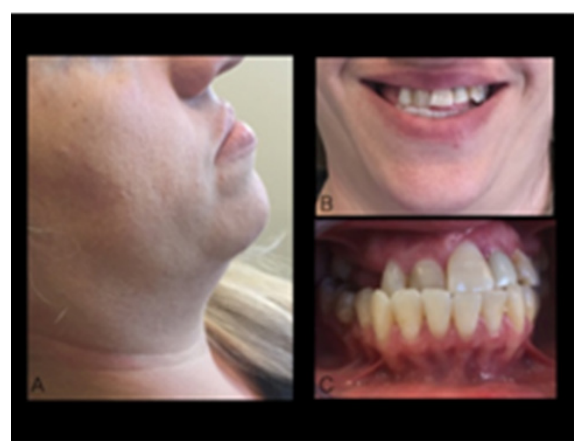

Figure I (A-C) Preoperative intra-oral clinical appearance. Note the abnormal transverse occlusal plane, deviation of the midline, maxillary atresia and class III malocclusion.

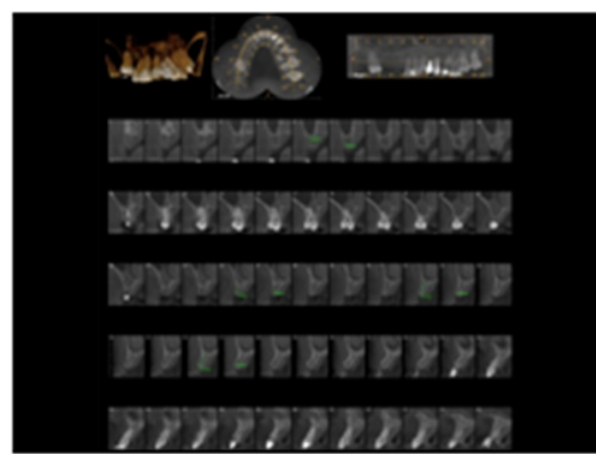

Figure 2 Preoperative $\mathrm{CBCT}$ and three-dimensional tomographic reconstruction for surgical planning. Note the root resorption of the anterior teeth.

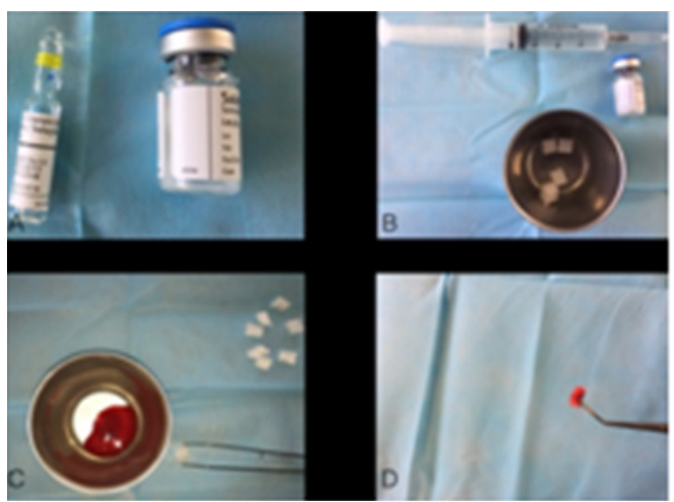

Figure 3 (A) RhGh; (B) Preparation of the material; (C\&D) Use of absorbable collagen sponge and patient blood to carry RhGh.

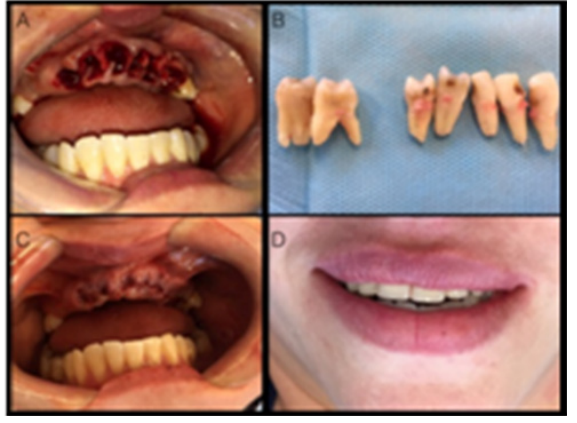

Figure 4 (A) Flapless and minimally invasive surgery aiming to preserve the buccal bone wall; (B) Dental elements extracted; (C) Suture prioritizing first intention healing. (D) Immediate removable partial denture performed to reestablish patient esthetic.

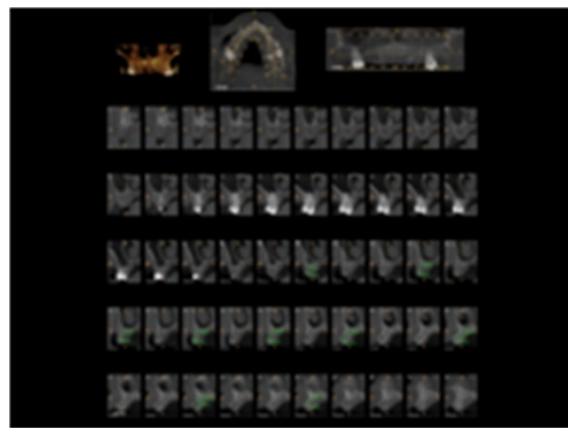

Figure $\mathbf{5}$ Immediate CBCT. Note all fresh sockets.

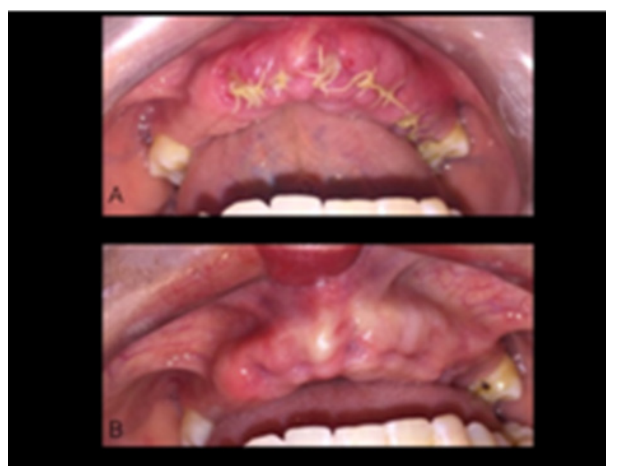

Figure 6 (A) Seven days follow-up. Good healing process, no signs of inflammation or infection; (B) Fourteen days of healing. Note the complete healing of the mucosa, with great buccal volume.

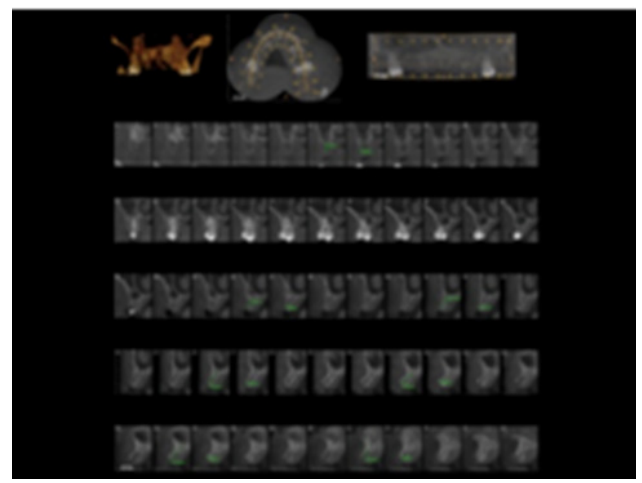

Figure $7 \mathrm{CBCT}$ after thirty days of healing. Note the preservation of the thin buccal bone wall. 


\section{Discussion}

Araujo \& Lindhe $\mathrm{J}^{12}$ compared minimally invasive surgery, without flap, with conventional surgery, in order to decrease the process of alveolar bone resorption. No significant differences were found between the benefit of the flapless surgery in relation to the alveolar reabsorption process after tooth extraction. Araujo \& Lindhe $\mathrm{J}^{13}$ stated that in humans, the application of different materials, such as alloplastic grafts, autogenous grafts, guided bone regeneration, xenogenic grafts and growth factors, aiming to maintaining the anatomical ridge dimensions post-extraction appear in studies with different success rates. Vignoletti et al. ${ }^{14}$ in a systematic review on surgical protocols for post-extraction alveolar preservation, categorized:

a. Clinical conditions of the alveolar site (integrity/non-integrity of alveolar bone walls, size and presence/absence of adjacent teeth).

b. Surgical protocol used (surgery with/without flap, first/second intention healing).

c. The biomaterial used (with/without membrane, biomaterial composition).

d. The type of evaluation used.

The results of this systematic review and meta-analysis showed that although some degree of bone resorption occur after tooth extraction, different alveolar preservation methods have resulted in a significant reduction in vertical and horizontal alveolar bone loss. The results obtained did not indicate the most indicated surgical approach or biomaterial, although with the use of membrane barriers, surgery with flap and total closure of the flap with first intention healing showed better results. Araújo et al. ${ }^{15}$ made a systematic review on alveolar bone healing and concluded that there are four main principles that should be taken into consideration in the alveolar bone healing process. First, the alveolus, in the anterior region of the maxilla, is characterized by a thin buccal bone wall. This thin wall promotes structure for the final vestibular contour after alveolar bone healing. Second, the buccal wall will be reabsorbed after tooth extraction. After bone resorption, the soft tissue collapses over the alveolus, creating an important bone defect. Third, immediate placement of implants does not prevent vestibular bone loss. In contrast, bone grafts limit the collapse of soft tissue over the alveolus and, at the same time, contribute in bone formation. Therefore, tooth extraction should be done with the understanding that alveolar bone crest reduction will occur and that some clinical approaches should be made to compensate this loss considering the future rehabilitation or replacement of the extracted tooth. Millis et al. ${ }^{9}$ evaluated the healing of osteotomies performed on the radio bone of Beagle dogs using somatropin (GW) at a dose of $2 \mathrm{mg} /$ day for 42 days. Promising results were found in the group that used somatropin in relation to the control group, having a scar radiographic evaluation superior to the control group, as well as bone density two to five times greater than the control group at the end of the experiment. According to literature, different bone grafting and alveolar ridge procedures are available. The application of different materials, such as alloplastic grafts, autogenous grafts, guided bone regeneration, xenogenic grafts and growth factors are predictable and suitable procedures, highly reported in many studies. The material used in this study has proved to be an alternative and effective strategy to increase tissue and bone healing after teeth extraction. After 3-4months healing and a new CBCT, it will be possible to have a better evaluation about the efficience of RhGh. Yang et al. ${ }^{11}$ made a systematic review of the literature on the use of Human Growth Hormone in hip fractures. They searched the databases Cochrane, PubMed, EMBASE, Chinese Bio-medicine database, China Journal Full-text Database and VIP database, where they selected 3 randomized clinical trials totaling 162 hip fractures comparing GH to Placebo. GH - treated fractures showed increased levels of insulin-like growth factor-1 (IGF-I) in the short term, but without significant differences over the long term. There was also no significant difference between groups in relation to adverse effects. As a conclusion the author suggests that GH may be effective in hip fractures. However, with the small number of participants and poor quality studies, it suggests that randomized, double-blind and placebo-controlled clinical trials are conducted to ensure this evidence. Eldein et al. ${ }^{7}$ evaluated the effect of Growth Hormone on dental implants performed in fresh alveolus. Six mutton dogs had their premolars extracted and immediate implants (control group) were performed on one side. On the opposite side, implants were performed with growth hormone in the implant bed, prior to its installation (test group). The animals were sacrificed at 2, 6 and 12 weeks after surgery. Histological sections were analyzed. At the end of the study, the authors conclude that the use of powdered GH around implants placed immediately after extractions considerably improve the peri-implant bone response. In conclusion, based on the literature, clinical and tomographic results of this case report, the RhGh could be an adequate material for use in alveolar fresh sockets. Despite of the results of this case, more studies are needed to evaluate whether the material is effective for preventing tissue collapses and maintaining the dimensions of the alveolar ridges, aiming a viable bone site for the placement of osseointegrated implants, in order to have a viable bone for rehabilitation.

\section{Acknowledgments}

None.

\section{Conflicts of interest}

There is not any conflict of interest.

\section{Funding}

None.

\section{References}

1. Kuboki Y, Hashimoto F, Ishibashi K. Time-dependent changes of collagen crosslinks in the socket after tooth extraction in rabbits. Journal of Dental Research. 1988;67(6):944-948.

2. Devlin H, Hoyland J, Newall JF, et al. Trabecular bone formation in the healing of the rodent molar tooth extraction socket. $J$ Bone and Miner Res. 1977;12(12):2061-2067.

3. Cardaropoli G, Araujo M, Lindhe J. Dynamics of bone tissue formation in tooth extraction sites. An experimental study in dogs. J Clin Periodontol. 2003;30(9):809-818.

4. Araujo MG, Lindhe J. Dimensional ridge alterations following tooth extraction: an experimental study in the dog. $J$ Clin Periodontol. 2005;32(2):212-218.

5. Fickl S, Zuhr O, Wachtel $\mathrm{H}$, et al. Tissue alterations after tooth extraction with and without surgical trauma: a volumetric study in the beagle dog. $J$ Clinical Periodontol. 2008;35(4):356-363. 
6. Tarnow DP, Eskow RN. Preservation of implant esthetics: soft tissue and restorative considerations. Journal of Esthetic Dentistry. 1996;8(6):12-19.

7. Eldein AMH, Elghamrawy SH, Osman SM, et al. Histological Evaluation of the Effect of Using Growth Hormone Around Immediate Dental Implants in Fresh Extraction Sockets: An Experimental Study. Implant Dent. 2018;20(1):47-55.

8. Isaksson OG, Janson JO, Gausse IA. Growth hormone stimulates longitudinal bone growth directly. Science. 1982;216(4551):1237-1239.

9. Millis DL, Wilkens BE, Daniel GB, et al. Radiographic, densitometric, and biomechanical effects of recombinant canine somatotropin in an unstable osteotomy gap model of bone healing in dogs. Vet Surg. 1998;27(2):85-93.

10. Guyton AC, Hall JE. Tratado De Fisiologia Médica. $9^{\text {th }}$ edn. Editora Guanabara Koogan S/A, Brazil. 1997. p. 1-1262.
11. Yang S, Cao 1, Cai S, et al. A systematic review of growth hormone for hip fractures. Growth Horm IGF Res. 2012;22(3-4):97-101.

12. Araujo MG, Lindhe J. Ridge alterations following tooth extraction with and without flap elevation: an experimental study in the dog. Clin Oral Implants Res. 2009;20(6):545-549.

13. Araujo MG, Lindhe J. Socket grafting with the use of autologous bone: an experimental study in the dog. Clin Oral Implants Res. 2011;22(1):9-13.

14. Vignoletti F, Matesanz P, Rodrigo D, et al. Surgical protocols for ridge preservation after tooth extraction. A systematic review. Clin Oral Implants Res. 2012;23(Suppl 5):22-38.

15. Araújo MG, Silva CO, Misawa M, et al. Alveolar socket healing: what we can learn? Periodontology. 2015;68(1):122-134. 